Philosophical Magazine,

Vol. ??, No. ?, Month?? 2005, 1-6

\title{
Structure of the pseudodecagonal Al-Co-Ni approximant PD4
}

\author{
P. OLEYNIKOV†, L. DEMCHENKO $\uparrow$, J. CHRISTENSEN $\uparrow$ t, \\ S. HOVMÖLLER $\uparrow$, T. YOKOSAWA $\uparrow$, M. DÖBLINGER $\downarrow$, \\ B. GRUSHKO§ and X. D. ZOU*† \\ $\dagger$ Structural Chemistry, Stockholm University, SE-106 91 Stockholm, Sweden \\ tDepartment of Chemistry and Biochemistry, \\ Ludwig-Maximilians-Universität München, D-81377 München, Germany \\ $\S I F F$, Forschungszentrum Jülich GmbH, D-52425 Jülich, Germany
}

(Received 15 May 2005; in final form 30 June 2005)

\begin{abstract}
A model for the pseudodecagonal approximant PD4 in the Al-Co-Ni system was deduced from single crystal X-ray diffraction data. The space group is $\mathrm{Bbmm}$ with $a=101.3, b=32.1$ and $c=4.1 \AA$. Atomic positions of 133 unique atoms in the unit cell with a reasonable geometry were found by direct methods and the difference Fourier syntheses. The obtained structure model is in good agreement with high-resolution electron microscopy images of PD4. Diffuse scattering observed along the $\boldsymbol{a}^{*}$ direction in the $h k l$ layers with $l=1 / 2,3 / 2$ etc. indicates a superstructure with a doubling of the periodicity along the $c$-axis and a lamellar disorder along the $\boldsymbol{a}$-axis. If this diffuse scattering is taken into account, $\boldsymbol{c}=8.2 \mathrm{~A}$.
\end{abstract}

\section{Introduction}

Since the discovery of quasicrystals (QCs) in 1984 [1], structural determination has been one of the most important issues in both their experimental and theoretical study. Quasicrystals display long-range atomic order, while lacking the periodicity of crystals. Many theoretical investigations of QC structures approximate the quasiperiodic structure by a sequence of periodic structures with growing unit cells [2-4]. For a number of QCs, there exist crystalline phases that resemble the quasicrystal, so-called approximant phases [2]. Approximants are classical crystals with a periodic structure, built up by the same clusters as in QCs, with diffraction patterns closely related to those of QCs. A whole hierarchy of approximant phases with growing unit cells should be expected.

Quasicrystals and their approximant phases have very similar chemical compositions, densities and electron diffraction patterns, and reversible transformations between the two occur [5-7]. This indicates that they have similar local structures. In the embedding picture, where the quasiperiodic tiling is embedded in a higherdimensional periodic lattice, approximants can be systematically constructed [8].

*Corresponding author. Email: zou@struc.su.se 
In practice, only the very first members of this hierarchy have been observed experimentally and, apart from a possibly tiny existence region, it might be hard to distinguish larger approximants from 'true' quasicrystals.

Thus, one approach to build the structures of quasicrystals is to understand the differences and relationships between quasicrystals and their approximant phases. So, crystalline approximants hold the key to determining the structures of quasicrystals.

In Al-Co-Ni and several other alloy systems, the periodic phases, approximants, are observed under certain conditions at the same compositions as corresponding QCs [9-11]. The term pseudodecagonal (PD) structure is used for decagonal QC approximants [12]. They are named PD1, PD2 etc. and exhibit diffraction patterns with almost perfect 10 -fold symmetry and quasiperiodic reflection arrangements of the strongest reflections [12-14].

As is well known, decagonal quasicrystals can be described by a quasiperiodic arrangement of clusters [15-18]. For example, all decagonal quasicrystals and highorder approximants in the $\mathrm{Al}-\mathrm{Ni}-\mathrm{Co}$ system comprise distinct 2-nm clusters [19-23]. The atomic arrangement within the clusters is imposing restrictions on the arrangement of the clusters themselves, as, for example, on overlapping of clusters [24-28]. A detailed determination of the atomic arrangements within the 2-nm clusters is important for understanding the structure of quasicrystal. Thus, we have undertaken this investigation.

\section{Experimental methods}

PD4 was obtained from an alloy of nominal composition $\mathrm{Al}_{72.5} \mathrm{Co}_{18} \mathrm{Ni}_{9.5}$ annealed at $900^{\circ} \mathrm{C}$ for $2150 \mathrm{~h}$ [12]. Single crystal X-ray diffraction (XRD) data was collected from a PD4 crystal on an Oxford Xcalibur ${ }^{\mathrm{TM}} 3$ diffractometer with monochromatized Mo $\mathrm{K} \alpha$ radiation.

The samples were also studied by electron diffraction and high-resolution electron microscopy (HREM) on a JEOL 3010 electron microscope operated at an accelerating voltage of $300 \mathrm{kV}$. Crystallographic image processing was performed using CRISP [29]. The local chemical composition was determined by energy dispersive spectroscopy (EDS) on a JEOL 2000FX electron microscope. HREM images and selected area electron diffraction (SAED) patterns were collected on powdered PD4 spread on $\mathrm{Cu}$ grids covered by holey carbon films.

\section{Results and discussion}

\subsection{Single-crystal $X$-ray and electron diffraction}

Reciprocal space reconstruction of the X-ray diffraction data performed by means of the CrysAlis RED program (Oxford Diffraction, 2002) indicates that PD4 is orthorhombic with a very large unit cell parameter along $a ; a=101.3, b=32.1$ and $c=4.1 \AA$ (figure 1). There are strong and sharp diffraction spots related to the 10-fold symmetry of decagonal QC. Weak and diffuse diffraction spots are observed at $l=1 / 2,3 / 2$, etc. This indicates that superstructure along the $c$-axis resulting in 

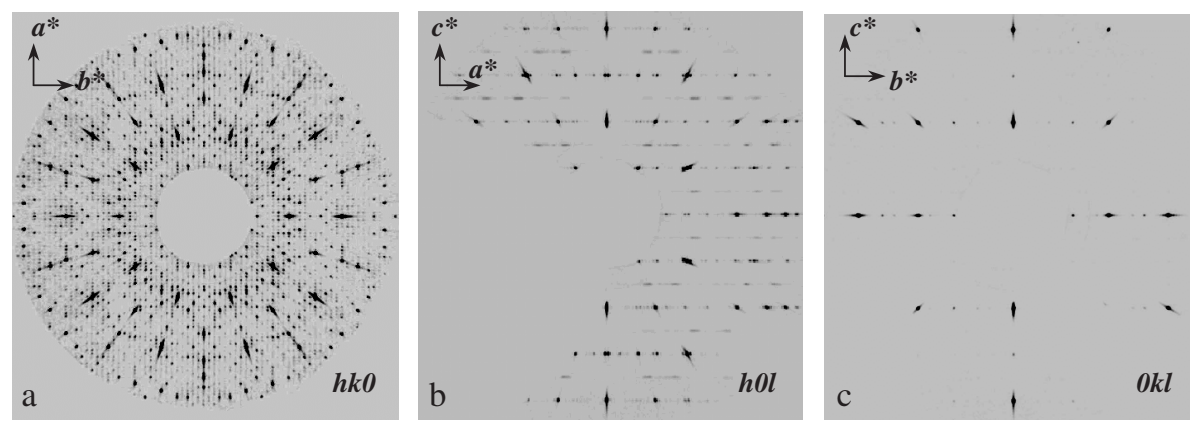

Figure 1. The (a) $h k 0$, (b) $h 0 l$ and (c) $0 k l$ layers of reciprocal space of PD4 from single crystal $\mathrm{X}$-ray diffraction. Note the pseudo- 10 -fold symmetry of the $h k 0$ pattern and the diffuse rows $(l=1 / 2 ; 3 / 2$, etc. $)$ in the $\boldsymbol{a}^{*}$ direction in the $h 0 l$ pattern.
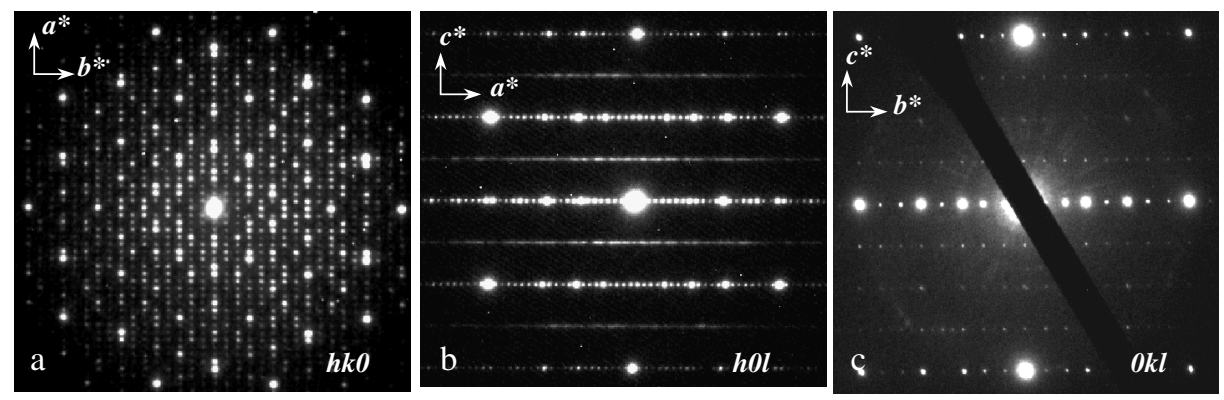

Figure 2. SAED patterns from PD4 taken along the $\boldsymbol{c}$-, $\boldsymbol{b}$ - and $\boldsymbol{a}$-axes. The magnification of these SAED patterns is twice that of the diffraction patterns in figure 1. Note the pseudo10 -fold symmetry of the $h k 0$ pattern and the diffuse rows $(l=1 / 2 ; 3 / 2$ etc. $)$ in the $\boldsymbol{a}^{*}$ direction in the $h 0 l$ pattern.

a doubling of the periodicity along the $c$-axis $(c=8.2 \AA)$. The diffuse streaking along the $\boldsymbol{a}^{*}$ direction (see reconstructed $h 0 l$ reciprocal lattice layer in figure 1 ) indicates a lamellar disorder along the $\boldsymbol{a}$-axis, which is observed frequently in the $\mathrm{Al}-\mathrm{Co}-\mathrm{Ni}$ system [30]. The selected area electron diffraction (SAED) patterns (figure 2) have some similarities to the X-ray diffraction patterns (figure 1). The same diffuse lines also clearly show up in the $h 0 l$ SAED patterns (figure 2). Since these diffuse layers only contribute less then $10 \%$ of the total scattered intensity, it was decided to first solve the main average structure, using $c=4.1 \AA$.

The intensities from single crystal XRD data were integrated and merged with the CrysAlis program. Lorentz and polarization corrections were applied and absorption effects were corrected. $h k l$ indices and intensities of reflections were extracted and the unit cell parameters calculated.

\subsection{Solving the structure and deducing an atomic model}

According to systematic absences $(h k l: h+l=2 \mathrm{n} ; h k 0: h=2 \mathrm{n} ; h 0 l: h+l=2 \mathrm{n} ; 0 \mathrm{kl}$ : $k=2 \mathrm{n}, l=2 \mathrm{n} ; h 00: h=2 \mathrm{n} ; 0 k 0: k=2 \mathrm{n} ; 00 l: l=2 \mathrm{n})$, the space group for PD4 was 
found to be Bbmm (no. 63). The average chemical composition of PD4 determined by EDS was virtually identical to the nominal composition.

A total of 35599 reflections, of which 5941 were unique, were collected. The PD4 initial structure model was deduced by direct methods in SIR2002 [31], using the 10000 strongest reflections (1818 unique). The program found 102 unique atoms. Successive difference Fourier syntheses using SHELXL97 [32] yielded 133 unique atomic positions in the unit cell with a reasonable geometry. Transition metal $(\mathrm{Co} / \mathrm{Ni})$ and aluminum positions were assigned according to the peak heights in the electron density map. The R-factor calculated by SHELXL97 for this model was 0.279 . Further determination of $\mathrm{Co}$ and $\mathrm{Ni}$ distribution in the unit cell is difficult due to the similarity of their X-ray scattering factors of these transition metals.

The strong reflections approach [33, 34] was also used to deduce a structure model, quite similar to that obtained by direct methods. The structure model of PD4 is shown in figure 3. The structure refinement is in progress.

\subsection{HREM}

HREM was applied in order to confirm the obtained model and to identify the clusters in our model. Figure 4 shows an experimental HREM image of PD4

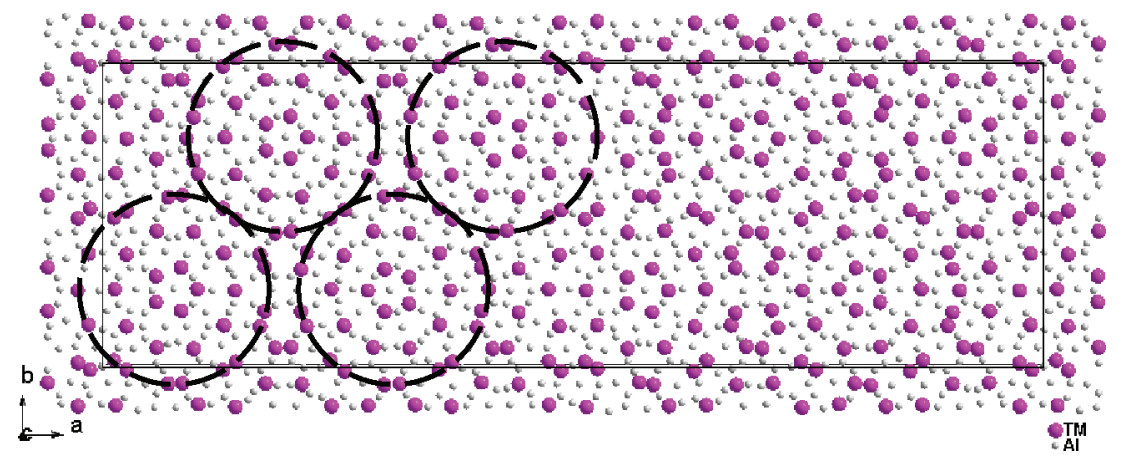

Figure 3. Structure model of PD4 projected along the $c$-axis. The circles indicate four of the eight 2-nm cluster columns in the unit cell. These clusters have pseudo-five-fold symmetry.

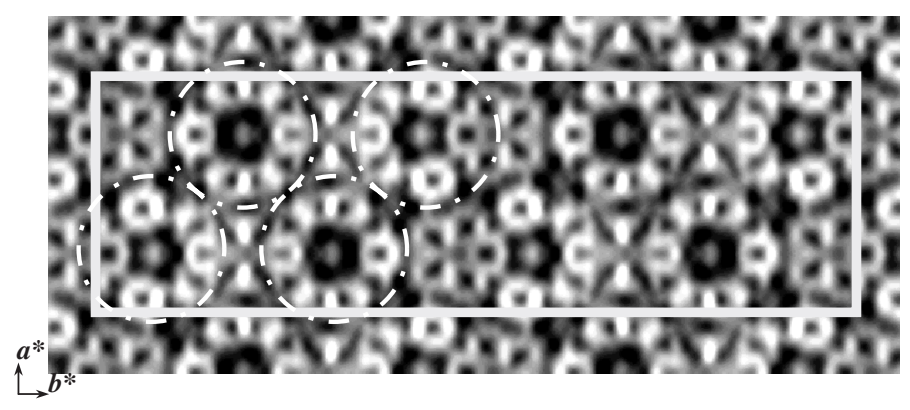

Figure 4. HREM image of PD4 taken along the pseudo-10-fold $c$-axis. The circles indicate the 2-nm cluster columns with pseudo-five-fold rotational symmetry. 


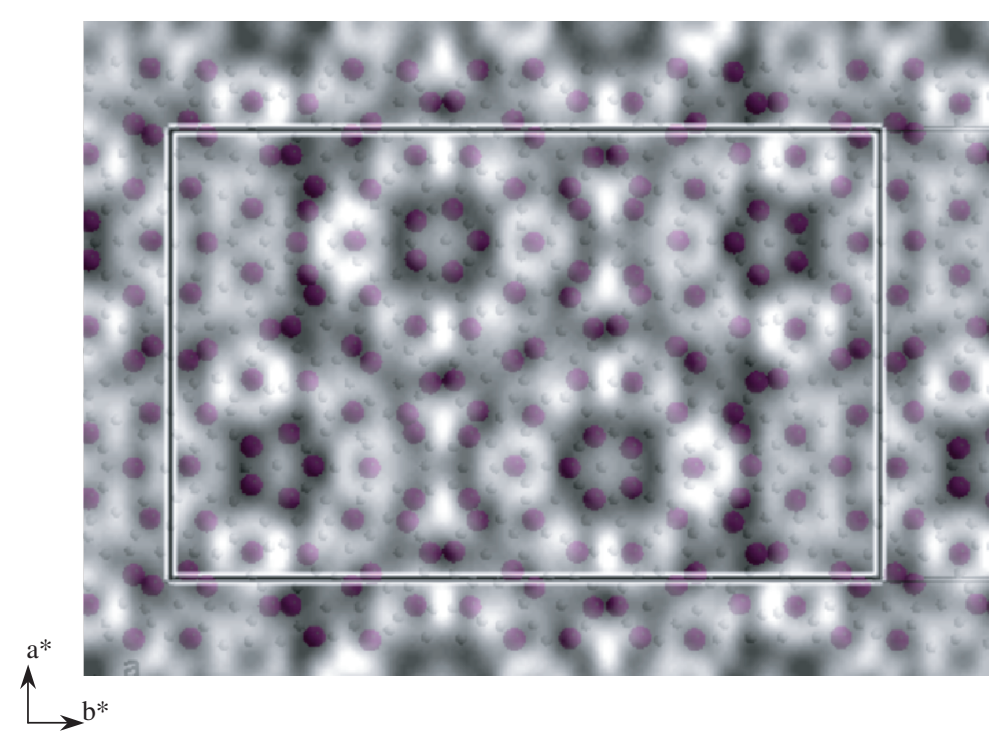

Figure 5. Atomic structure obtained by X-rays overlapped on a HREM image projected along the short $\boldsymbol{c}$-axis. Due to the $B$-centering, the projected unit cell is halved along the $\boldsymbol{a}$ axis (becoming $50.6 \AA$ ). $\mathrm{Ni} / \mathrm{Co}$ atoms are in dark, and $\mathrm{Al}$ atoms are in grey. Large dots are atoms at the $z=0$ layer, small dots are atoms at $z=0.5$.

taken along the $c$-axis after crystallographic image processing was applied. The $(h k)$ projection symmetry was determined from HREM images using CRISP [29]. The plane group for PD4 was found to be $p m g$ with the mirror plane $m$ perpendicular to the $\boldsymbol{b}$-axis. Most of the projected unit cell is covered by circular clusters, $2 \mathrm{~nm}$ in diameter (indicated in figure 4). The structure model obtained by X-rays agrees well with the HREM images as seen in figure 5. Dark spots belonging to Co and $\mathrm{Ni}$ atoms appear around the perimeters of each of the 2-nm cluster columns and in two more circular arrangements, with diameters of 13.3 and $6.5 \AA$, respectively. Five aluminum atoms were reassigned to be transition metal atoms and three transition metal atoms were reassigned to be aluminum. The 2-nm cluster columns have approximate five-fold rotational symmetry.

\section{Conclusion}

A structure model of PD4 has been obtained from single crystal X-ray diffraction and electron microscopy. The atomic arrangement corresponding to the 2-nm cluster column with 5-fold rotational symmetry for PD4 was found.

\section{Acknowledgements}

This project is supported by the Swedish Research Council. X. D. Zou is a research fellow of the Royal Swedish Academy of Sciences supported by a grant from the 
Knut and Alice Wallenberg Foundation. L. Demchenko is supported by a grant from the Royal Swedish Academy of Sciences.

\section{References}

[1] D. Shechtman, I. Blech, D. Gratias, et al., Phys. Rev. Lett. 531951 (1984).

[2] A.I. Goldman and K.F. Kelton, Rev. Mod. Phys. 65213 (1993).

[3] J.W. Cahn, D. Gratias and D. Shechtman, Nature 319102 (1986).

[4] M. Duneau, R. Mosseri and C. Oguey, J. Phys. A 224549 (1989).

[5] P.A. Bancel, Phys. Rev. Lett. 632741 (1989).

[6] E.R. Abe and A.P. Tsai, Phys. Rev. Lett. 83753 (1999).

[7] X.D. Zou, K.K. Fung and K.H. Kuo, Phys. Rev. B 354526 (1987).

[8] M. Baake, D. Joseph and P. Kramer, J. Phys. A 24 L961 (1991).

[9] J. Grin, U. Burkhardt, M. Ellner, et al., J. Alloys Compd. 206243 (1994).

[10] C. Freiburg, B. Grushko, R. Wittenberg, et al., Mater. Sci. Forum 228/231 583 (1996).

[11] X.L. Ma and K.H. Kuo, Metall. Trans. A 231121 (1992).

[12] B. Grushko, D. Holland-Moritz and R. Wittmann, et al., J. Alloys Compd. 280 215 (1998).

[13] B. Grushko, D. Holland-Moritz and K. Bickmann, J. Alloys Compd. 236243 (1996).

[14] B. Grushko, M. Döblinger, R. Wittmann, et al., J. Alloys Comp. 34230 (2002).

[15] P. Guyot and M. Audier, Phil. Mag. B 52 L15 (1985).

[16] V. Elserand and C.L. Henley, Phys. Rev. Lett. 552883 (1985).

[17] W. Steurer, Acta Crystallogr. B 45534 (1989).

[18] H.-C. Jeong and S.J. Steinhardt, Phys. Rev. Lett. 731943 (1994).

[19] K. Hiraga, F.J. Lincoln and W. Sun, Mater. Trans. JIM 32308 (1991).

[20] K. Saitoh, K. Tsuda, M. Tanaka, et al., Phil. Mag. A 73387 (1996).

[21] K. Saitoh, K. Tsuda, M. Tanaka, et al., Jpn. J. Appl. Phys. 36 L1400 (1998).

[22] K. Tsuda, Y. Nishida, K. Saitoh, et al., Phil. Mag. A 74697 (1996).

[23] S. Ritsch, C. Beeli, H.-U. Nissen, et al., Phil. Mag. Lett. 7499 (1996).

[24] W. Steurer and K.H. Kuo, Acta Crystallogr. B 46703 (1990).

[25] S.E. Burkov, Phys. Rev. Lett. 67614 (1991).

[26] A. Yamamoto, Sci. Rep. Res. Inst. Tohoku Univ. A 42207 (1996).

[27] K. Saitoh, K. Tsuda and M. Tanaka, Phil. Mag. A 76135 (1997).

[28] E. Cockayne and M. Widom, Phys. Rev. Lett. 81598 (1998).

[29] S. Hovmöller, Ultramicroscopy 41121 (1992).

[30] F. Frey, in Fundamental Materials Science Series, edited by M.F. Thorpe and S.J.L. Billinge (Plenum Press, New York, 1998), pp. 295-321.

[31] M.C. Burla, M. Camalli, B. Carrozzini, et al., J. Appl. Crystallogr. 361103 (2003).

[32] G.M. Sheldrick, SHELX-97. Program for Crystal Structure Determination (University of Göttingen, Germany, 1994).

[33] J. Christensen, P. Oleynikov, S. Hovmöller, et al., Ferroelectrics 305273 (2004).

[34] H. Zhang, X.D. Zou, P. Oleynikov, et al., Phil. Mag. B submitted (2005). 


\section{AUTHOR QUERIES}

\section{Journal id: TPHM $-\mathbf{1 2 7 0 1 7}$}

Query

number

\section{Query}

1 Oxford diffraction 2002--missing in reference list. Please advise

$2 \quad$ Please update reference 34 